\title{
Endocrine Disruptor Compounds (EDC) Effects on Reproduction
}

\author{
Thi Mong Diep Nguyen \\ Faculty of Biology-Agricultural Engineering, Quy Nhon University, Quy Nhon, Viet Nam
}

Email address:

diepdhqn@ymail.com

\section{To cite this article:}

Thi Mong Diep Nguyen. Endocrine Disruptor Compounds (EDC) Effects on Reproduction. International Journal of Diabetes and Endocrinology. Vol. 3, No. 1, 2018, pp. 18-22. doi: 10.11648/j.ijde.20180301.14

Received: March 25, 2018; Accepted: April 12, 2018; Published: May 14, 2018

\begin{abstract}
A large number of industrial chemical products (paints, flame retardants, pharmaceuticals, pesticides) are suspected or proved to act as endocrine disruptor compounds (EDCs). In the present survey of the literature, the main issue is to consider how to classify individual products as EDC or not. In this perspective, the different mechanisms leading to endocrine disruption are described: either by direct interaction with hormone receptors leading to its stimulation or inhibition, or by effect on endogenous hormone concentration through stimulation or inhibition of its synthesis or its degradation, or its binding to transport binding proteins. The present review takes particularly in consideration the effects of EDCs on the endocrine control of Reproduction in human and animals. Indeed, a number of EDCs can act as anti-androgens, anti-estrogens, and steroidogenic enzyme inhibitors that interfere with steroid action or production and thus can alter reproductive health.
\end{abstract}

Keywords: Endocrine Disruption, Endogenous Hormone, Reproduction, Reproductive Health

\section{Introduction}

Many chemicals have been shown or suspected to exhibit endocrine disruption activity. This means that these endocrine disruptor compounds (EDCs) can, in various ways, interfere with the endocrine system of animals. EDCs potentially interfere with the production, secretion, metabolism, transport or peripheral action of endogenous hormones by means of their binding to hormone receptors. These substances display a variety of idiosyncratic biochemical effects, with complex and multifactorial mechanisms of disruption of endocrine systems. In the present paper the functional characteristics of endocrine disruptors are defined in order to evaluate if their toxicity toward non-target species is primarily due to endocrine disruption. Particular emphasis is put on the reproductive system of vertebrates as this function is primordial in the survival and evolution of animal species. In this scope it is nevertheless important to differentiate endocrine disruption from reprotoxicity directly affecting reproduction steps (for example gametes production or survival).

The main mechanisms of action of EDCs are described as well as their main impacts on the reproductive function in vertebrates. The cocktail effect and non-monotonous dose- response effects are also discussed in relation with differences between EDCs and hormones.

\section{Mechanisms of Action of EDCs}

Drugs and their metabolites are a source of possible or proved EDCs. Their ED activity is a consequence of their normal therapeutic action considered as an EDC when peoples other than those taking them to cure their illness are unintentionally exposed to it (presence of these drugs or their metabolites in drinking water for example). Similarly, pesticides that are synthetic molecules not aiming at curing, but at being toxic towards fungi, plants or animals detrimental to cultures. Nevertheless, these pesticides can be toxic to Human and wild fauna. Other proved or suspected EDCs are not aimed at curing or destroying living beings (paints, antifowling coatings, cans coatings, medical tubings) but their structures lead them to interfere with the endocrine system.

Endocrine Disruptor Compounds (EDCs) are molecules, natural or synthetic, that interfere with the endocrine network of vertebrates, provoking adverse dysregulation of the hormonally-controlled physiological parameters or functions [1], including reproduction. EDCs include persistent pesticides and herbicides, methoxychlor, biocides, heat stabilizers, and chemical catalysts, plastic contaminants, 
pharmaceuticals, or dietary components. Those exogenous compounds can arise from industrial and domestic effluents or agricultural and urban runoff. The general population continues to be exposed to EDCs through ingestion of contaminated food, inhalation of contaminated air and dust, and skin contact. This interference can occur through different mechanisms [2]: either directly by binding to the hormone receptor, or indirectly by increasing or decreasing the concentration of available active hormone (s).

The most direct mechanism is an interaction of the EDCs with an hormone receptor leading either to stimulation [3] or inhibition of downstream cellular pathway in target cells. Figure 1 shows this type of mechanism where an exogenous molecule can bind to the hormone receptor. Doing so this molecule can either inhibit hormone action or stimulate by itself the receptor activation pathway. Usually, only nuclear receptors are the targets of such direct action by EDCs i.e. the receptors for steroid hormones (progesterone, estrogens, testosterone, glucocorticoids, mineralocorticoids), for thyroid hormones (thyroxine), for vitamin D (calcitriol), for cis- or trans-retinoic acids, for PPAR and also a number of orphan receptors.

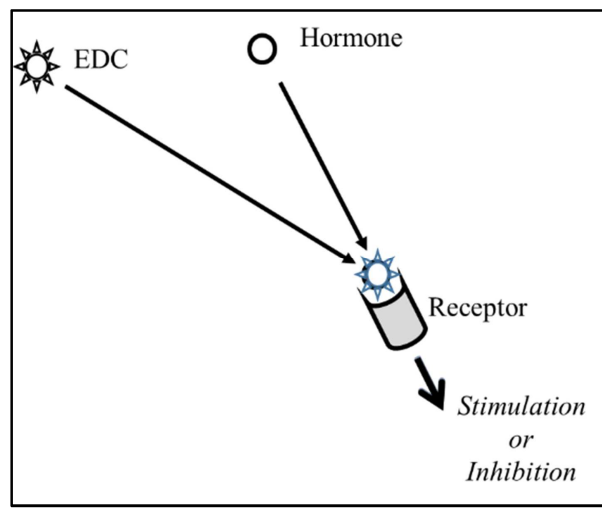

Figure 1. Scheme of endocrine disruptor direct interaction with a hormone receptor leading to indue receptor stimulation or to inhibition of hormone action on its receptor.

The second type of mechanism is through modification of endogenous hormone concentrations [3-4], by stimulation or inhibition of either their synthesis (Figure 2) or degradation (Figure 3).

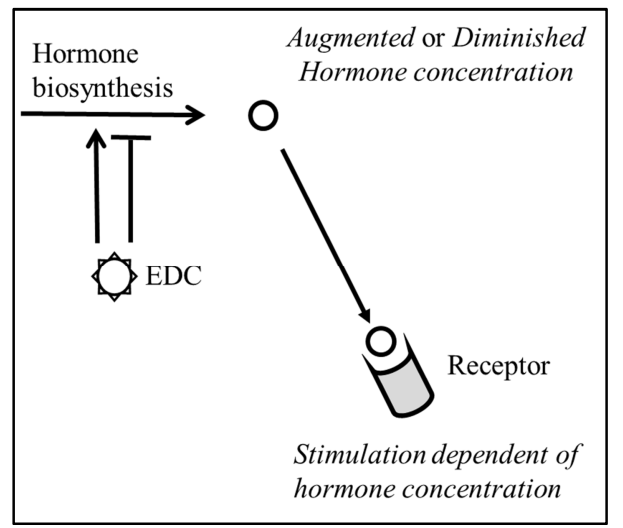

Figure 2. Scheme of endocrine disruptor indirect action through stimulation or inhibition of a hormone biosynthesis.

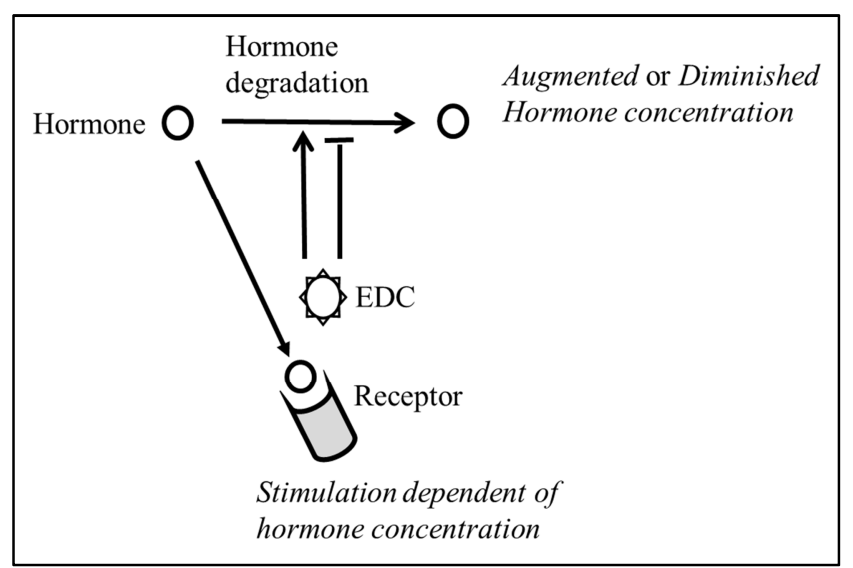

Figure 3. Scheme of endocrine disruptor indirect action through stimulation or inhibition of a hormone degradation or elimination from blood circulation.

Finally, interference by EDCs can also occur through modulation of hormone-binding proteins concentrations that control the availability of active hormones (Figure 4).

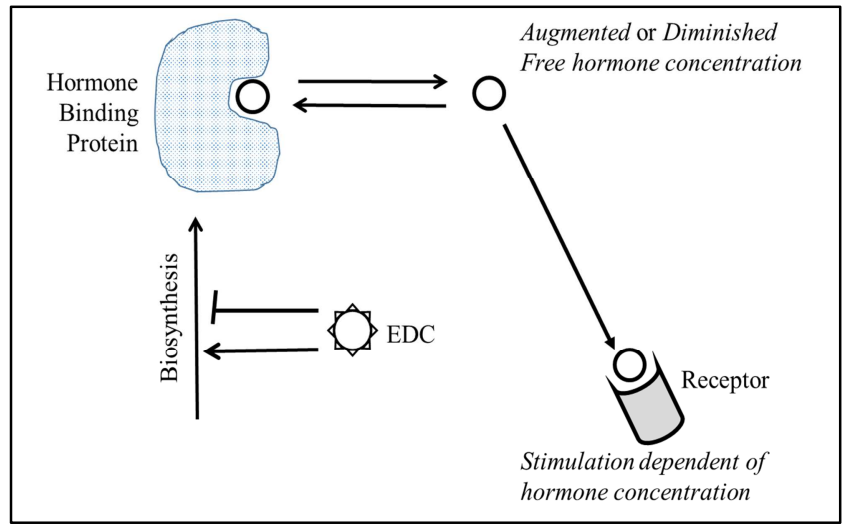

Figure 4. Scheme of endocrine disruptor indirect action through stimulation or inhibition of a hormone-binding protein leading modification of free active hormone concentration in blood.

A number of in vitro and in vivo tests have been set up by diverse national and international agencies to identify EDCs [4-7]. Only the first mechanism (direct action on a receptor) can be easily tested in vitro using either soluble receptors or cells cultures expressing the receptor in a functional state. The three other mechanisms can only be evidenced in in vivo tests.

\section{Endocrine Networks and Hypothalamus-Pituitary-Gonads (HPG) Axis}

The endocrine system is constituted by several networks of hormones, allowing the coordinate functions of dozens of different cell types. Each network possesses numerous loops of stimulation and retroaction in cascade so that the different physiological parameters (such as glycemia, lipidemia, hydro-mineral balances, etc) and physiological functions (such as development, growth, immunity, reproduction, etc.) 
are set in the proper range for the good health of the whole organism and for the survival of species. In this paper, my aim is to introduce a number of central questions concerning EDCs with particular interest in vertebrate reproduction.

The general control of reproduction in vertebrates occur through HPG axis that can be the target of EDCs at different levels [8-11]. Also the hypothalamo-pituitary-adrenocortical (HPA) axis can impact on mammalian male and female reproduction and development of offspring [12] as will be detailed below.

\section{Examples of Endocrine Disruption Affecting Reproduction}

In males, it has been found that a number of recognized EDCs can affect steroidogenesis and sperm production in adults but also affect normal testis, seminiferous ducts or brain development [13]. In females EDCs exposure can lead to precocious puberty, polycystic ovary syndrome, and premature ovarian failure.

There are many examples of direct interaction of xenobiotics with nuclear receptors, particularly the estrogen receptor. It is puzzling that bisphenol A (BPA) that has been used for years in plastic bottles and cans, had been initially developed for contraceptive pills. It was thus not a surprise later to detect its estrogenic activity that, nevertheless, is 100 000-fold less than that of ethinyl-estradiol, the currently most used estrogen in contraceptive pills. Because of its estrogenic activity, BPA has been banned first from use in plastic bottles for babies and in many countries from any alimentary packaging. The effects of this type of EDCs is due to their ability to accommodate the receptor binding pocket for hormone. Upon binding, the EDC can promote a change of receptor conformation that leads either to its activation or its inhibition (Figure 1). Of course, the EDCs that activate the estrogen receptor exhibit lowering of fertility in females.

As shown in Figure 2, endocrine disruption can occur through impact on hormone synthesis. For example, stress in rats suppresses testosterone secretion via a direct inhibitory effect of elevated glucocorticoids on Leydig cells. A number of xenobiotics have been shown to affect $11 \beta$-HSD1 (reductase) and 11 $\beta$-HSD2 (oxidase) that play important roles in glucocorticoid synthesis. Therefore, they can act as EDCs through their influence on glucocorticoid circulating concentrations. Through their direct effect they can impact fetal development and through their effect on testicular testosterone, they can impact male reproduction.

The mechanisms shown in figures $3 \& 4$ are much more difficult to detect but should not be discarded in efforts to understand the different ways of EDCs actions.

\section{What Make Endocrine Disruptors Different from Hormones}

Hormones and their receptors have co-evolved as to ensure proper specificity of hormone-receptor pairs in all generations of evolving and diversifying species. The natural ligands for nuclear receptors are not polypeptide or protein hormones and therefore, they are not directly encoded by genes. It can also be observed that the hormones binding to nuclear receptors are very well conserved during evolution (estrogens, progestagens, thyroid hormones etc.). It can thus be concluded that the evolution of nuclear receptors has been restricted by the need for recognizing its cognate hormone and, also by the need NOT to bind another circulating molecule. It can be hypothesized that an animal with a receptor allowing its stimulation by a wrong circulating ligand would not survive or would not be able to reproduce (which is the same in term of Evolution).

The problem with the arrival of thousands of small industrial organic molecules is that the nuclear receptors have not been selected during Evolution to avoid their binding. Therefore, a certain percentage of these molecules can, more or less, accommodate the receptors binding pocket and promote receptor activation or inactivation; i.e. these molecules act as endocrine disruptors. Moreover, conjugating and degrading systems have also been set up during Evolution to limit the half-life of hormones whereas synthetic molecules can remain unchanged and potentially accumulate in the body.

\section{Hazard Versus Risk of Endocrine-Disruptors}

As for all other forms of toxicity, it is important to consider both the potential hazard of all suspected molecules as thoroughly as possible in order to evaluate the risk for human and/or wildlife populations. In this prospect, it is important to consider $1 /$ exposure of populations to the chemical under study, $2 /$ the dose-responses of its effects and 3 / the cocktail effect.

In the case of pesticides, it is clear that the professional exposure [14] is considerably higher than the general population exposure but the former can take appropriate measures, if correctly informed while the latter should not be exposed to detrimental levels of pesticide (s). Moreover, the prenatal and postnatal periods of development until puberty are particularly sensitive because of their persistent effects due to possible detrimental effects during developmental steps [15]. Exposure of the general population to persistent organic pollutants, including pesticides, can be followed by detection of their presence in body fluids or tissues. Due to the extreme sensitivity of most modern assays, it is necessary to consider not solely the presence of these molecules, but their actual concentration.

As for all other forms of toxicity, potency of potential endocrine disruptors is an important information to consider and the risk is generally dependent on both exposure and potency.

Non-monotonous dose-responses are sometimes claimed as a hallmark of endocrine disruptor effects. The observation in some experiments, of U-shape dose-response curves where low doses of chemical exhibit higher effect than medium doses, and comparable effect with high doses cannot be 
attributed to intrinsic properties of endocrine disruptors. Indeed, it is rather due to the complexity of endocrine regulations in animals and depends on the chosen end-point for the study of the effect (s). The EDCs exhibit low affinity and low specificity towards their target-proteins. Indeed, they generally bind to one or several proteins (receptors, binding proteins, conjugating enzymes etc...) susceptible to affect animals' endocrine system. Their positive and negative effects at different sites can lead to non-monotonous responses, eventually different depending on the endpoint chosen. Moreover, the view that it is a property of EDCs to exhibit higher activity at lower doses than at higher doses, could lead to the erroneous simplistic opinion that it would better to always have a certain level of EDCs in the environment to avoid the low-doses effects.

It is clear that the number of potentially toxic molecules in the environment leads to take in consideration the possibly additive, or worse, synergic effects of EDCs. Owing to the complexity of endocrine regulations, such effects would require to study EDCs two by two or more. It is of course an impossible task, and rather, representative molecules with given mechanisms should be studied for additive, synergic or counteracting effects with representative molecules of other groups to detect the most likely cocktail effects.

\section{Cocktail Effect}

When several endocrine disruptors are present together (cocktail), it is expected that they exhibit either additive or synergic effects. It is extremely difficult to tackle this problem because all mixtures at all concentrations cannot be studied. It is thus necessary to make theoretical assumptions and select mathematical models that greatly influence the final conclusion.

In a recent study [16] an organotypic culture system of human fetal testes explants was used to screen eleven selected compounds individually or as mixtures of 4 to 8 . Their effects as antiandrogens were tested and analyzed. No clear cut conclusion is drawn by the authors but our interpretation is that the effects are additive rather than synergic.

It must be stressed here that this study was performed in vitro, i.e. in a simplified system compared to in vivo. Due to the multiplicity of cell interactions in a human or animal body, much more complex interactions between endocrine disruptors can occur.

Classification of molecules under study per mechanism (s) of action permits to take into account potential additive [1718], synergistic or counteracting cocktail effects [18-19].

\section{Conclusion}

Endocrine disruption is a mild but insidious form of toxicity so that it is often difficult to prove or reject its involvement. The complexity of endocrine regulations in vertebrates together with the high number of new synthetic molecules render the task of determining the endocrine disrupting risk for each molecule, enormous and often contradictory. For these reasons classification of molecules under study per mechanism (s) of action on endocrine regulations would be helpful to reduce the number of assays needed to validate endocrine disruption.

Concerning Reproduction, there are many steps that can be sensitive to potential endocrine disruptors. The most sensitive periods to EDCs during lifetime are the fetal and perinatal periods as well as puberty during which proper regulations must be set for permitting good fertility at adulthood.

\section{References}

[1] De Falco M, Sellitti A, Sciarrillo R, Capaldo A, Valiante S, Iachetta G, Forte M, Laforgia V (2014) Nonylphenol effects on the HPA axis of the bioindicator vertebrate, Podarcis sicula lizard. Chemosphere 104: 190-196.

[2] Combarnous Y (2017) Endocrine Disruptor Compounds (EDCs) and agriculture: The case of pesticides. C R Biol 340: 406-409.

[3] Golub M, Doherty J (2004) Triphenyltin as a potential human endocrine disruptor. $J$ Toxicol Environ Health B Crit Rev 7: 281-295.

[4] Bishop PL, Willett CE (2014) The use and acceptance of Other Scientifically Relevant Information (OSRI) in the U. S. Environmental Protection Agency (EPA) Endocrine Disruptor Screening Program. Birth Defects Res B Dev Reprod Toxicol 101: 3-22.

[5] Willett CE, Bishop PL, Sullivan KM (2011) Application of an integrated testing strategy to the U. S. EPA endocrine disruptor screening program. Toxicol Sci 123: 15-25.

[6] Borgert CJ, Mihaich EM, Quill TF, Marty MS, Levine SL, Becker RA (2011) Evaluation of EPA's Tier 1 Endocrine Screening Battery and recommendations for improving the interpretation of screening results. Regul Toxicol Pharmacol 59: 397-411.

[7] Reif DM, Martin MT, Tan SW, Houck KA, Judson RS, Richard AM, Knudsen TB, Dix DJ, Kavlock RJ (2010) Endocrine profiling and prioritization of environmental chemicals using ToxCast data. Environ Health Perspect 118: 1714-1720.

[8] Yin P, Li YW, Chen QL, Liu ZH (2017) Diethylstilbestrol, flutamide and their combination impaired the spermatogenesis of male adult zebrafish through disrupting HPG axis, meiosis and apoptosis. Aquat Toxicol 185: 129-137.

[9] Kariyazono Y, Taura J, Hattori Y, Ishii Y, Narimatsu S, Fujimura M, Takeda T, Yamada H (2015) Effect of in utero exposure to endocrine disruptors on fetal steroidogenesis governed by the pituitary-gonad axis: a study in rats using different ways of administration. J Toxicol Sci 40: 909-916.

[10] Sikka SC, Wang R (2008) Endocrine disruptors and estrogenic effects on male reproductive axis. Asian J Androl 10: 134-145.

[11] Warita K, Sugawara T, Yue ZP, Tsukahara S, Mutoh K, Hasegawa Y, Kitagawa H, Mori C, Hoshi N (2006) Progression of the dose-related effects of estrogenic endocrine disruptors, an important factor in declining fertility, differs between the hypothalamo-pituitary axis and reproductive organs of male mice. J Vet Med Sci 68: 1257-1267. 
[12] Witorsch RJ (2016) Effects of elevated glucocorticoids on reproduction and development: relevance to endocrine disruptor screening. Crit Rev Toxicol 46: 420-436.

[13] Jeng HA (2014) Exposure to endocrine disrupting chemicals and male reproductive health. Front Public Health 2: 55.

[14] Blanco-Munoz J, Lacasana M, Lopez-Flores I, RodriguezBarranco M, Gonzalez-Alzaga B, Bassol S, Cebrian ME, Lopez-Carrillo L, Aguilar-Garduno C (2016) Association between organochlorine pesticide exposure and thyroid hormones in floriculture workers. Environ Res 150: 357-363.

[15] Goldman LR, Koduru S (2000) Chemicals in the environment and developmental toxicity to children: a public health and policy perspective. Environ Health Perspect 108 Suppl 3: 443-448.

[16] Gaudriault P, Mazaud-Guittot S, Lavoue V, Coiffec I, Lesne L, Dejucq-Rainsford N, Scholze M, Kortenkamp A, Jegou B (2017) Endocrine Disruption in Human Fetal Testis Explants by Individual and Combined Exposures to Selected Pharmaceuticals, Pesticides, and Environmental Pollutants. Environ Health Perspect 125: 087004.
[17] de Sousa G, Nawaz A, Cravedi JP, Rahmani R (2014) A concentration addition model to assess activation of the pregnane $\mathrm{X}$ receptor (PXR) by pesticide mixtures found in the French diet. Toxicol Sci 141: 234-243.

[18] Crepet A, Heraud F, Bechaux C, Gouze ME, Pierlot S, Fastier A, Leblanc J, Le Hegarat L, Takakura N, Fessard V, Tressou J, Maximilien R, de Sousa G, Nawaz A, Zucchini-Pascal N, Rahmani R, Audebert M, Graillot V, Cravedi JP (2013) The PERICLES research program: an integrated approach to characterize the combined effects of mixtures of pesticide residues to which the French population is exposed. Toxicology 313: 83-93.

[19] Ahmed MA, Vogel CF, Matsumura F (2015) Unique biochemical and molecular biological mechanism of synergistic actions of formamidine compounds on selected pyrethroid and neonicotinoid insecticides on the fourth instar larvae of Aedes aegypti (Diptera: Culicidae). Pestic Biochem Physiol 120: 57-63. 